\title{
Simulating video-assisted thoracoscopic lobectomy: A virtual reality cognitive task simulation
}

\author{
Brian Solomon, MD, Costas Bizekis, MD, Sophia L. Dellis, BA, Jessica S. Donington, MD, \\ Aaron Oliker, MSc, Leora B. Balsam, MD, Michael Zervos, MD, Aubrey C. Galloway, MD, \\ Harvey Pass, MD, and Eugene A. Grossi, MD
}

\begin{abstract}
Objective: Current video-assisted thoracoscopic surgery training models rely on animals or mannequins to teach procedural skills. These approaches lack inherent teaching/testing capability and are limited by cost, anatomic variations, and single use. In response, we hypothesized that video-assisted thoracoscopic surgery right upper lobe resection could be simulated in a virtual reality environment with commercial software.
\end{abstract}

\begin{abstract}
Methods: An anatomy explorer (Maya [Autodesk Inc, San Rafael, Calif] models of the chest and hilar structures) and simulation engine were adapted. Design goals included freedom of port placement, incorporation of well-known anatomic variants, teaching and testing modes, haptic feedback for the dissection, ability to perform the anatomic divisions, and a portable platform.

Results: Preexisting commercial models did not provide sufficient surgical detail, and extensive modeling modifications were required. Video-assisted thoracoscopic surgery right upper lobe resection simulation is initiated with a random vein and artery variation. The trainee proceeds in a teaching or testing mode. A knowledge database currently includes 13 anatomic identifications and 20 high-yield lung cancer learning points. The "patient" is presented in the left lateral decubitus position. After initial camera port placement, the endoscopic view is displayed and the thoracoscope is manipulated via the haptic device. The thoracoscope port can be relocated; additional ports are placed using an external "operating room" view. Unrestricted endoscopic exploration of the thorax is allowed. An endo-dissector tool allows for hilar dissection, and a virtual stapling device divides structures. The trainee's performance is reported.
\end{abstract}

Conclusions: A virtual reality cognitive task simulation can overcome the deficiencies of existing training models. Performance scoring is being validated as we assess this simulator for cognitive and technical surgical education. (J Thorac Cardiovasc Surg 2011;141:249-55)

Video clip is available online.

Virtual reality (VR) has become an increasingly popular modality of surgical education in recent years. Useful VR simulators are cost-effective, high-yield training modalities that can integrate 3-dimensional imaging, customizable instrumentation, and haptic feedback. These simulators have

\footnotetext{
From the Department of Cardiothoracic Surgery, New York University School of Medicine, New York, NY.

This work was partially funded by a Simulation in Thoracic Surgery Education Award (to E.A.G.) from the Thoracic Surgery Foundation for Research and Education. Disclosures: Authors have nothing to disclose with regard to commercial support. Presented at the 36th Annual Meeting of The Western Thoracic Surgical Association, Ojai, California, June 23-26, 2010.

Jessica S. Donington is a member of The Western Thoracic Surgical Association. Received for publication June 18, 2010; revisions received Aug 5, 2010; accepted for publication Sept 9, 2010.

Address for reprints: Eugene A. Grossi, MD, NYU Medical Center, Suite 9-V, 530 First Avenue, New York, NY 10016 (E-mail: grossi@cv.med.nyu.edu). $0022-5223 / \$ 36.00$

Copyright (C) 2011 Published by Elsevier Inc. on behalf of The American Association for Thoracic Surgery

doi:10.1016/j.jtcvs.2010.09.014
}

a high initial development cost but can effectively eliminate the ongoing costs of cadaver or animal tissue models. Furthermore, these simulators have the capacity to expose trainees to a multitude of anatomic variations and integrate didactic lectures, videos, and assessments. Such simulators also eliminate the need for continuous one-on-one instructor observation by electronically monitoring progress. Simulators have the added benefit of providing the opportunity for unlimited repetitions to optimize the user's skill through practice, without destroying tissue or exposing patients to risk.

We hypothesized that video-assisted thoracoscopic surgery (VATS) right upper lobe (VRUL) resection could be "performed" in a VR environment with commercially available software. By using and expanding on a known virtual surgery simulation platform (BioDigital Systems, LLC, New York, NY), we developed the design structure for a low-cost, widely distributable simulator aimed at teaching the anatomy and techniques necessary to perform a VRUL resection to medical trainees, residents, fellows, and practicing surgeons. The simulator teaches basic surgical skills, spatial relations, anatomic approaches, and cognitive knowledge associated with VRUL resection on a PC-based 


\section{Abbreviations and Acronyms \\ VATS = video-assisted thoracoscopic surgery \\ $\mathrm{VR}=$ virtual reality \\ VRCTS $=$ virtual reality cognitive task simulation \\ VRUL $=$ VATS right upper lobe}

platform. This simulator provides a dynamic 3-dimensional teaching and testing tool for cognitive surgical knowledge. The simulator mimics a complete surgical resection in an engaging, real-time, interactive environment. The user is able to navigate through all the steps of a complex surgical procedure. The simulator also provides an interactive testing mode that incorporates feedback mechanisms and simulated error pathways. This computerized VR platform presents a novel opportunity to standardize, teach, and test procedural knowledge in thoracic surgery. This article describes the strategies used to create such a state-of-the-art tool.

\section{MATERIALS AND METHODS}

\section{Technical Components and Platform}

The simulator system includes a standard "gaming" laptop PC (Dell, Austin, Tex) and a haptic feedback device (Sensable Omni, Cambridge, Mass) used to control the surgical instruments. The haptic device handle allows trainees to touch and manipulate the virtual objects seen on the screen. The handle is mounted in a restrictive mechanical chest wall that mimics the physical constraints of a standard VATS port site. The simulation software is based on a VR biomedical visualization engine specifically developed for anatomic education by BioDigital Systems, LLC. This system incorporates 3-dimensional animation and virtual training environments and stores scientific data. The system displays highly detailed 3-dimensional models of thoracic anatomy from any chosen perspective. Specifically, this system has been developed to display a 3-dimensional surgical operative environment in real-time from both external and thoracoscopic perspectives.

The capacity to "teach or test" didactic information associated with the anatomy and surgical procedure is integrated into the software engine. This provides feedback and instills error pathways, improving the trainee's cognitive performance by allowing the trainee to experience failure and success.

\section{Simulation}

Graphic models of the chest, right lung, hilar structures, and mediastinum were created in Maya (Autodesk Inc, San Rafael, Calif) and loaded into the simulation engine. Design goals included (1) freedom of port placement for camera and instruments; (2) accurate anatomic representation, incorporating common anatomic variants; (3) teaching and testing modes; (4) haptic feedback for the dissection of hilar structures; (5) ability to perform the anatomic divisions necessary for a VRUL resection; and (6) a portable PC-based platform to host the simulation. Design methodology focused on reducing VRUL resection into a series of steps. Four VR instruments were developed and integrated into the surgical environment (Table 1). The successive use of these instruments allows the trainee to perform the fundamental steps required in VRUL resection (Video 1). Throughout the simulation, the trainee is presented with essential learning points related to VRUL resection, including identification of anatomy, physiologic principles, potential complications, and surgical methodology (Appendix 1). Afterward, the trainee is provided with an assessment of surgical performance and achievement of educational goals.

\section{RESULTS}

Preexisting commercial anatomic models did not provide sufficient surgical detail, and extensive modeling modifications were necessary. VRUL simulation begins with a random combination of predefined hilar vein and artery anatomic variations, and the trainee proceeds in a teaching or testing mode. The educational database currently includes 12 anatomic identifications and 18 high-yield lung cancer learning points (Appendix 1). The "patient" is presented in the left lateral decubitus position (Figure 1). After an initial camera port is chosen, the endoscopic camera view is displayed, and the thoracoscope is manipulated via the haptic input device. The thoracoscope port can be relocated at any time, and additional ports can be placed using an external "operating room" view. Unrestricted endoscopic exploration of the thorax is allowed, with the ability to manipulate the lung into 3 predefined positions, mimicking standard intraoperative retraction. A virtual endo-dissector can be used for hilar dissection, and a virtual stapling device can be used to divide the hilar structures. At the end of the simulation, the trainee's performance is reported.

\section{Simulated Procedural Flow}

When the program starts, various anatomic variations and anomalies are randomized and loaded to present a unique surgical experience for each operation. Current models include 3 different pulmonary vein variations, 2 pulmonary artery variations, and the presence/absence of pleural metastatic disease (Figure 2). The trainee is presented with a screen in which a "picture-in-picture" insert demonstrates the patient in a posterolateral thoracotomy position. The trainee then has to use a mouse to choose a location on the chest wall for a port site and camera insertion. After port placement, the main screen shows the camera view of a 30degree thoracoscope. Manipulation of the camera position is performed via the haptic handle. The trainee is expected to inspect and identify the anatomy and prepare for insertion of a second port. If the trainee has chosen a "poor" location for the camera port, the camera port can be removed and the trainee then can choose a new port position.

The current model does not allow for operator distraction of the lung; this is currently accomplished via a menu toggle. The initial position is neutral, and the operator has the choice of whether to deflect the lung anteriorly or posteriorly to provide the exposure necessary for the particular hilar dissection being performed.

The trainee/operator inserts another port for an endodissecting tool. This is haptically enabled to allow the operator to dissect ("rub away") pleura and lung parenchyma to expose the anatomy of the hilum (Figure 3). At this point, the operator is expected to identify the specific anatomic variant and determine the appropriate points for vessel and bronchial ligation. Once the areas for ligation and 
TABLE 1. Simulated instrumentation

\begin{tabular}{lc}
\hline \multicolumn{1}{c}{ Instrument } & Functionality \\
\hline $\begin{array}{l}\text { Trocar/port } \\
\text { Thoracoscope (30 degree) }\end{array}$ & $\begin{array}{c}\text { Allows trainee to choose from multiple sites of chest entry } \\
\text { Provides visualization of the thoracic cavity, limited by the physical restraints } \\
\text { of trocar placement in the chest wall and intrathoracic structures (collision detection) }\end{array}$ \\
$\begin{array}{l}\text { Tissue endo-dissector } \\
\text { Stapler }\end{array}$ & $\begin{array}{l}\text { Via haptic tissue contact allows "dissection" to expose underlying hilar structures } \\
\text { Viact allows division of predefined segments of hilar anatomy }\end{array}$ \\
\hline
\end{tabular}

division have been identified, a third port (for a stapling device) is inserted. Contact by the endostapler with segmental anatomic structures allows for multiple (correct and incorrect) divisions of the branch veins, arteries, and bronchus in the hilum (Figure 4). Throughout this procedure, the operator must shift the lung and camera position to provide the appropriate thoracoscopic view of the structures being dissected and divided. In doing so, the operator is able to perform a complete right upper lobe resection.

The software is designed to identify common errors in procedural flow, including tears in pulmonary parenchyma that would result in air leaks, inappropriate ligation of vessels/bronchi too close to the pulmonary hilar origin, ligation of the vessels of the right middle or lower lobe, and failure to ligate vessels to the right upper lobe. Throughout the procedure, the operator is able to dissect regional lymph nodes. This feature educates the operator on the anatomic borders of each lymph node level and the appropriate approach to dissect each nodal station. Specific focuses of the simulator include lymph node levels $2 \mathrm{R}, 4 \mathrm{R}$, and 7 .

An additional feature built into the system is the "anatomy explorer." This feature allows the operator to navigate through the anatomy of the right chest without performing the resection. The operators can turn on/off specific components of the anatomy, dissect the anatomy, and label each portion of the anatomy.

Finally, a testing mode has been built into this educational system. This mode enables instructive questions to be inserted into the procedure in a window to the right of the resection screen. In this mode, specific questions will be presented as "pop-ups" at key points throughout the procedure. As these questions arise, the simulation can be paused to allow the operator to answer each question. The simulator tracks answers and allows the operator to review explanations for any question answered incorrectly. These questions test the operator's knowledge of anatomy, procedural steps, and pulmonary pathophysiology.

\section{DISCUSSION}

The traditional apprenticeship model of thoracic surgical education, which was once only limited by resident stamina, is now limited by increasing concerns for patient safety, expanding procedural complexity and diversity, and resident work-hour restrictions. Despite this, recent analyses predict that complex endoscopic procedures may require 25 to 100 repetitions to develop competency and safety. ${ }^{1,2}$ To

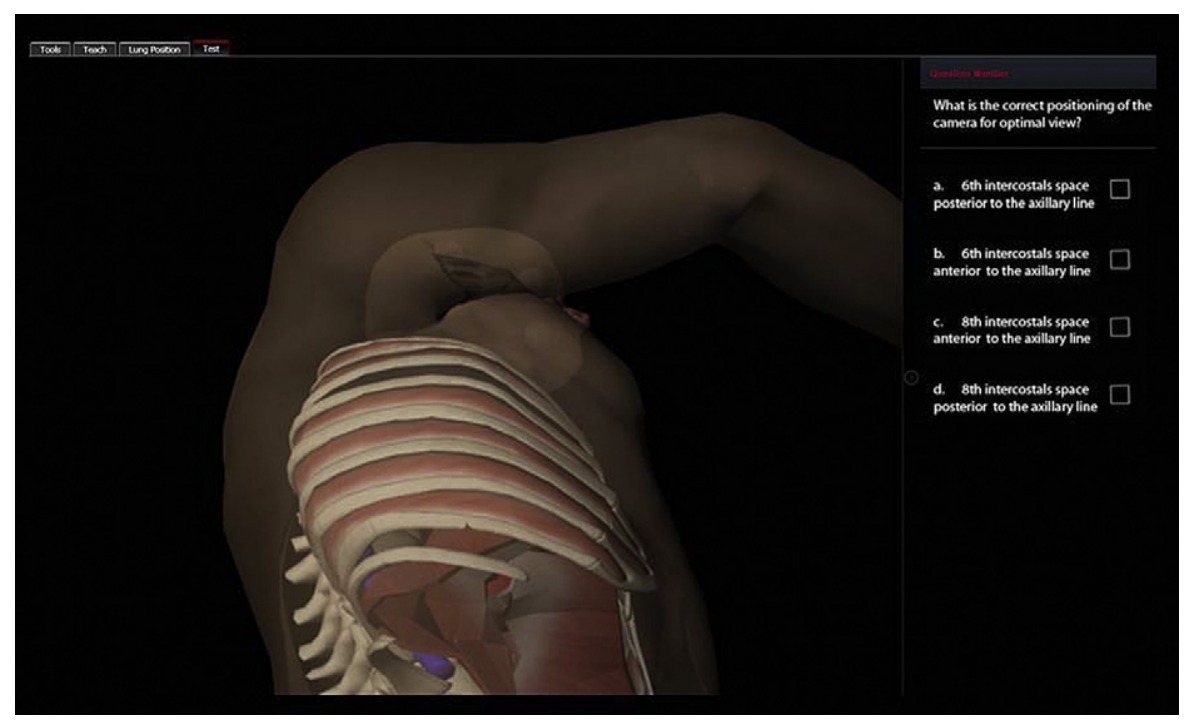

FIGURE 1. Initial simulation approach showing the patient in the left lateral decubitus position with the right arm extended to expose the right chest. The trainee is now ready to select a site for trocar and thoracoscope insertion. The image also shows the integration of didactic education and testing built into the simulation platform. 


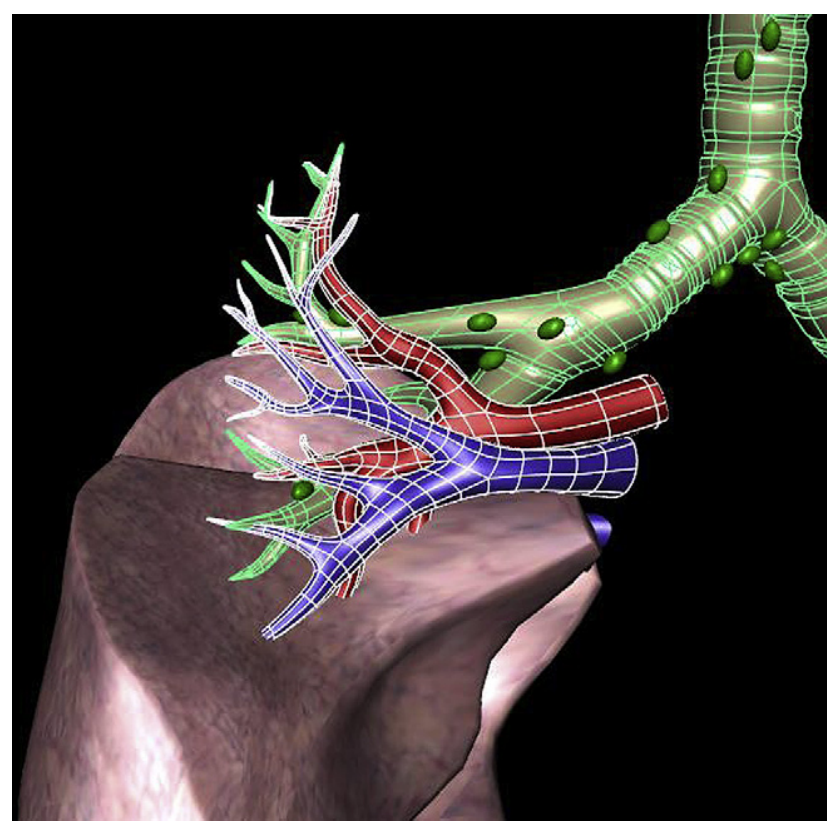

FIGURE 2. Segmented underlying pulmonary vasculature and bronchial anatomy built into the system. The most common anatomic variant of pulmonary anatomy is demonstrated.

reconcile these factors, thoracic surgery educators are currently looking toward other educational models, including simulation, to improve cognitive and procedural skills before reaching the operating room. The optimal methodology for such simulation-based training and assessment is a current subject of extensive investigation and debate.

Cognitive simulators designed to challenge both cognitive and psychomotor resources provide an important basis for effective training and evaluation. ${ }^{3}$ The interaction of the cognitive and psychomotor layers in simulations has been demonstrated to enhance the learning process. ${ }^{3}$ The presented VR cognitive task simulation (VRCTS) combines 3-dimensional task training (psychomotor) with didactic education and an assessment process (cognition). This simulation is an extension of other pivotal cognitive simulators, such as the plastic surgery simulator described by Cutting and colleagues. ${ }^{4}$ By using a limited 3-dimensional VR simulation in cleft lip and palate surgery, these investigators demonstrated educational effectiveness in their "Smiletrain" project. This VRUL project expands on that model by incorporating a psychomotor component.

A paucity of simulators for complex thoracic surgery procedures has been described. Carter and Marshall ${ }^{5}$ developed a high-fidelity open lobectomy model using a fiberglass chest wall model and a prepared bovine lung. Trainees received didactic training on the simulator and then performed a lobectomy under the guidance of a thoracic surgeon. A knowledge-based exam was administered and an objective structured assessment of technical skill (OSATS) scale rating ${ }^{6}$ was used to assess procedural com-

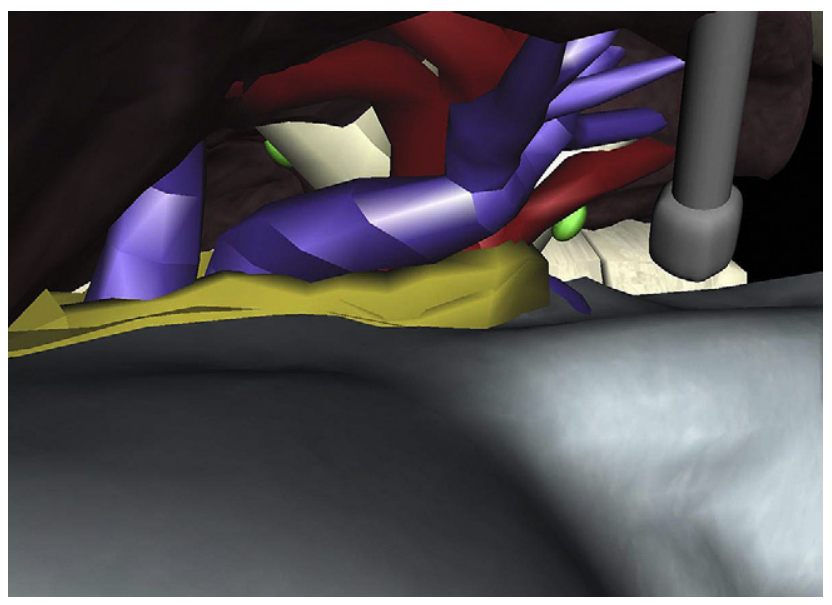

FIGURE 3. Use of the endo-dissection tool for mobilizing the lung tissue and exposing underlying vasculature.

petency. Repetitive use demonstrated improvement in scores; the impact on operative performance was not evaluated. Although the methodology is effective at procedural teaching, it is limited by the cost and single use of animal tissue and the need for a thoracic surgeon to be present for each iteration.

We are seeking additional grant support so multiple additional modules can be developed for this platform. Comparable to the low-end prices of currently available VR surgical simulators, an enhanced version of this simulator should cost in the range of $\$ 25,000$ to $\$ 35,000$ per unit, and commercial distribution should be available in the next 1 to 2 years.

Our VRCTS is a hybrid between low- and high-fidelity models. The low-fidelity component teaches the dissection involved in thoracoscopic surgery, whereas the high-fidelity component integrates thoracic knowledge base, thoracoscopic skills, 3-dimensional anatomy, and variable anatomy models to teach specific components related to the procedure. Prior simulations have relied largely on integrated 3-dimension animations, but this simulator also integrates haptic feedback and 3-dimensional dissection to further enrich the educational experience. This model shares design features with another successful VR simulator, the Shoulder and Knee "Arthro VR Virtual Reality" trainer (GMV, Madrid, Spain), which uses 3-dimensional VR technology and haptic feedback to train orthopedic residents to perform arthroscopic surgery. Howells and colleagues ${ }^{7}$ found improved performance in the operating room (better transfer of motion psychomotor skills, based on the Orthopaedic Competence Assessment Project score and the global rating scale) in residents trained on the simulator.

Accurate training simulators have the potential for broadbased use in surgical education. In general surgery, the Fundamentals of Laparoscopic Surgery is a successful simulation training program that is widely integrated in general 


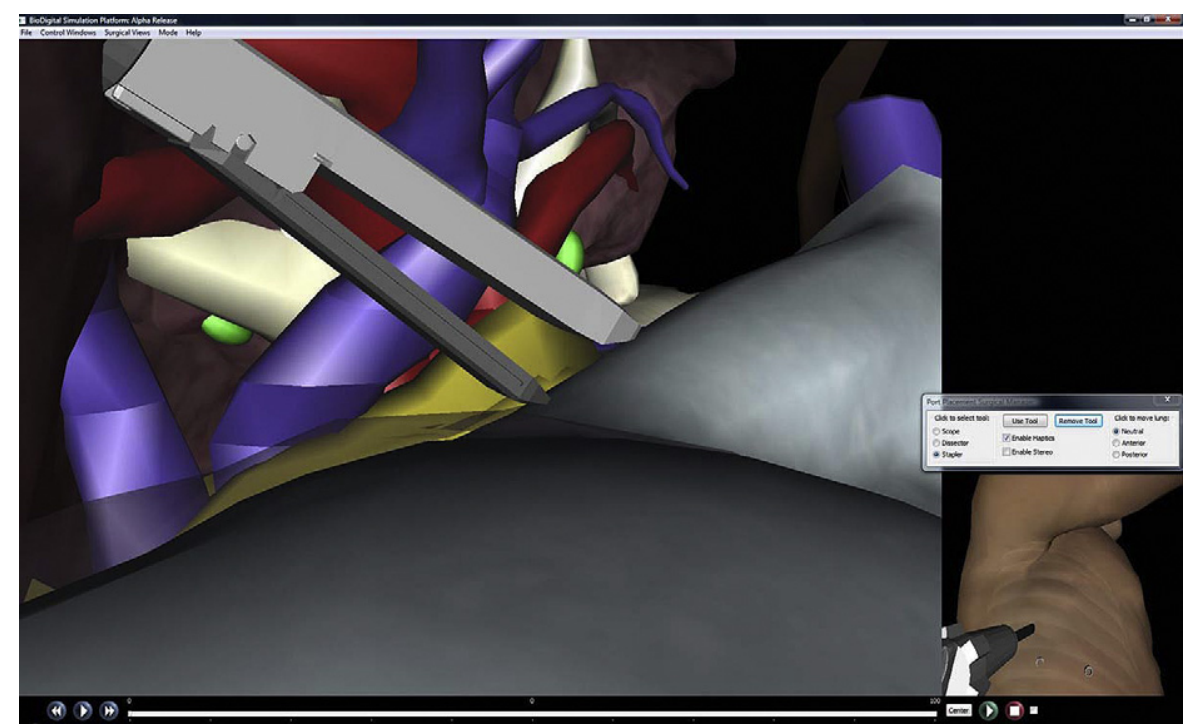

FIGURE 4. Use of the thoracoscopic stapler for dividing the pulmonary vasculature and bronchial tissue.

surgery residency training programs. ${ }^{8}$ This program, which is devoid of specific anatomic information, teaches both cognitive and declarative knowledge and psychomotor skills. Multiple studies have shown that performance is improved through the use of simulated training. In vitro laparoscopic simulation practice directly correlates with improved cognitive skills and performance on operative and in vivo animal models. ${ }^{9,10}$ The Fundamentals of Laparoscopic Surgery program has been validated by rigorous metrics. ${ }^{8}$

It is now recognized that VR training can improve surgical competency. The Minimally Invasive Surgical TrainerVirtual Reality system was evaluated in a randomized, double-blinded study that demonstrated faster dissection, decreased risk of harm to the gallbladder, and fewer surgical errors by residents trained on the system. ${ }^{11}$ We believe that VRCTS technology is appearing at a critical time in thoracic surgery education, given the ongoing reform of our residency programs.

The stakeholders in this education process are not only the residents but also the program directors, the surgical boards, and the patients. The advent of "integrated" training programs for thoracic residents has enhanced the need for such training and evaluation tools because a "final" binary assessment at the end of a 6-year period is not practical. Training and evaluation tools, including simulators, provide feedback and assessment at earlier and more appropriate times in the training program. ${ }^{12}$ Integrating and mandating cognitive task simulators into the training process also could lead to the use of these simulators as an auxiliary tool for the maintenance of certification.

\section{Limitations}

Current limitations of the model include the haptic sensation being limited to gross dissection. Fine motor function and physics-based tissue response has not yet been built into the simulator. Therefore, although users are able to "dissect" simulated lung tissue, they are not yet capable of dissecting the hilar vessels with fine precision or the haptics associated with "going around" a vessel to isolate it. The simulator is also limited by the number of initial camera port locations and by predefined mobilization of the lung positions. This simulator does not yet have patient-specific anatomy incorporated; further simulation platform development will be necessary to "import" patient datasets. Although the current version is aimed at a single user, the addition of a second haptic device would provide the potential for team training for the surgical procedure.

\section{CONCLUSIONS}

Our VRUL resection VR task trainer can help overcome deficiencies of existing training models and provide important experience for the operator. Performance scoring is currently being validated and is the subject of our ongoing research. Once validated, the model will be readily expandable to other pulmonary resections, as well as other procedures in cardiac and esophageal surgery.

\section{References}

1. Schauer P, Ikramuddin S, Hamad G, Gourash W. The learning curve for laparoscopic Roux-en-Y gastric bypass is 100 cases. Surg Endosc. 2003;17:212-5.

2. Hawasli A, Lloyd LR. Laparoscopic cholecystectomy. The learning curve: report of 50 patients. Am Surg. 1991;57:542-5.

3. Kahol K, Vankipuram M, Smith ML. Cognitive simulators for medical education and training. J Biomed Inform. 2009;42:593-604

4. Cutting C, Oliker A, Haring J, Dayan J, Smith D. Use of three-dimensional computer graphic animation to illustrate cleft lip and palate surgery. Comput Aided Surg. 2002;7:326-31

5. Carter YM, Marshall MB. Open lobectomy simulator is an effective tool for teaching thoracic surgical skills. Ann Thorac Surg. 2009;87:1546-51.

6. Martin JA, Regehr G, Reznick R, et al. Objective structured assessment of technical skill (OSATS) for surgical residents. Br J Surg. 1997;84:273-8. 
7. Howells NR, Gill HS, Carr AJ, Price AJ, Rees JL. Transferring simulated arthroscopic skills to the operating theatre: a randomised blinded study. J Bone Joint Surg Br. 2008;90:494-9.

8. Peters JH, Fried GM, Swanstrom LL, et al. Development and validation of a comprehensive program of education and assessment of the basic fundamentals of laparoscopic surgery. Surgery. 2004;135:21-7.

9. Fried GM, Derossis AM, Bothwell J, Sigman HH. Comparison of laparoscopic performance in vivo with performance measured in a laparoscopic simulator. Surg Endosc. 1999;13:1077-82.

10. Derossis AM, Fried GM, Abrahamowicz M, Sigman HH, Barkun JS, Meakins JL. Development of a model for training and evaluation of laparoscopic skills. Am J Surg. 1998;175:482-7.

11. Seymour NE, Gallagher AG, Roman SA, et al. Virtual reality training improves operating room performance: results of a randomized, double-blinded study. Ann Surg. 2002;236:458-64.

12. Scott DJ, Ritter EM, Tesfay ST, Pimentel EA, Nagji A, Fried GM. Certification pass rate of $100 \%$ for fundamentals of laparoscopic surgery skills after proficiency-based training. Surg Endosc. 2008;22:1887-93.

\section{APPENDIX 1. Integrated anatomic identification in right upper lobe resection simulator}

1. Pulmonary hilum

2. Superior pulmonary vein

3. Pulmonary artery

4. Bronchus

5. Azygous vein

6. Esophagus

7. Right upper lobe bronchus

8. Middle lobe vein and variants

9. Lymph nodes (zone 4)

10. Lymph nodes (zone 7)

11. Inferior pulmonary ligament

12. Recurrent superior segment artery

13. Right upper lobe vein

\section{TEACHING POINTS}

1. Selection of appropriate patients for VATS lobectomy.

2. Surgical indications and contraindications for VATS lobectomy.

3. Relevance of specific pulmonary function tests for stratification of risk factors for VRUL resection.

4. Appropriate preoperative anesthesia preparation, including placement of double-lumen endotracheal tube.

5. The correct camera positioning is the eighth intercostal space anterior to the axillary line.

6. The correct positioning of the anterior instrument port site is above the horizontal fissure (usually in the fourth to fifth intercostal space).

7. The correct positioning of the posterior instrument port site is above the dome of the diaphragm (usually posterior axillary line eighth to ninth intercostal space).

8. The anatomic landmark separating the right upper lobe from the right middle lobe is the horizontal (minor) fissure.

9. The anatomic landmark separating the right upper and middle lobes from the right lower lobe is the major fissure.
10. Appropriate positioning of the lung to identify and dissect pulmonary vasculature.

11. Appropriate positioning of the lung to identify and dissect bronchial tissue.

12. The right upper lobe consists of apical, anterior, and posterior segments.

13. The superior pulmonary vein is the most anterior structure in the pulmonary hilum.

14. The apical anterior trunk is directly posterior to the superior pulmonary vein.

15. The double layer of pleura connecting the lower lobe to the mediastinum and the medial part of the diaphragm is the inferior pulmonary ligament.

16. Appropriate procedure for identification of air leaks intraoperatively.

17. Identification of lymph nodes stations $4 \mathrm{R}$ and 7 , including anatomic borders.

\section{Discussion}

Dr Richard Whyte (Stanford, Calif). Surgical education is changing, and there is increased emphasis on education, systems-based practice, safety, core competencies, work-hour restrictions, and so on, and less on the "service" component of a resident's job. Furthermore, we are getting away from an apprenticeship model and the old "see one, do one, teach one" approach. One aspect of this change is that of simulation. It has been going on for some time. David Gaba, at our own institution, has developed an anesthesia simulator and has been using it for approximately 15 years to teach residents how to manage crises in the operating room. And of course the airline industry has been doing this for a long time; the downside there is that, although the simulators are of high fidelity, they cost $\$ 20$ million or so, a little outside of our reach. Surgery has been somewhat slow to start on this but certainly is catching up, and this article is an excellent example of where things are going. To follow on this, the Thoracic Surgery Foundation for Research and Education, as you know, has put $\$ 100,000$ into surgical simulation, specifically thoracic, and I think you and your colleagues are the benefactors of this to a certain extent. Furthermore, I think it is important that the federal government emphasize surgical simulation. A number of us spent a couple of days last week in Washington, DC, advocating for passage of the Enhancing Simulation Act of 2009, which would allocate approximately $\$ 50$ million in 2010 to medical simulation in general, and I would hope that surgery would be part of this.

Minimally invasive surgery provides new and fertile ground for simulation-based training, and this article is an excellent example of where things are going. The model is not perfect, but no models are. Certainly it has some advantages in terms of being able to describe anatomic variants and allowing the student to do this over and over again and improve their skills. As to disadvantages, I think there is probably a lack of tactile feedback, but this is an area where there will be progress. The costs of this simulation technology is probably somewhat high and is something that would be interesting to hear about, but my 2 questions are how will you and your colleagues specifically put this into the educational format or syllabus for your trainees, how do you coordinate it with pig models or operating room-based training, and how do you plan 
to translate this across to other institutions so they can take advantage of some of these novel technologies?

Dr Solomon. To answer the first question regarding integration of this into the curriculum, we have currently written a manual that we are working on distributing to the residents, and it will be integrated into the New York University simulation laboratory. The plan will be to get residents to spend time to repeat multiple iterations. We have not yet determined the validated measure that we are going to use to measure whether residents are ready to go to the operating room and perform these procedures. We have noticed in all prior studies that residents have improved with repeated iterations, so the goal would be to monitor these residents over time and see how they are improving on the simulator. As I mentioned, this is a low-fidelity simulator. It is meant mainly to teach the cognitive points of the simulation and the basic anatomic spatial relations and basic dissection. It does not teach the high-fidelity portions of getting around vessels, so obviously it will be integrated with pig models and other models to teach the actual sewing vessels and getting around vessels. Both will be integrated into the curriculum, and hopefully the long-term goal for validation will be to train residents on it and then test them in the operating room and see how their performance improves in the operating room with time and with training.

Regarding your second question, one of our goals was to make this as portable and easily distributable of a system as possible. The entire system fits into a single small suitcase. It is 1 or will be expandable to 2 robotic arms and a single laptop computer, so it is easily sharable with other universities, with other programs, and hopefully in the thoracic boot camp model we can bring it down there and train residents initially with the simulator.

Dr Donald Low (Seattle, Wash). I believe Dr Whyte also asked you about cost, and I will add to that: Is this program now mandatory for residents to use or are you still testing as to where it is going to ultimately fit?

Dr Solomon. We are still testing as to where it is going to fit. We are hoping to make it mandatory within the coming years.

Regarding the cost, there is a large initial cost of developing this. As you mentioned, the airline industry has $\$ 20$ million grants to develop their simulators, and they create high-fidelity simulators for flying jets. We do not have anywhere near that kind of money, but we are hoping that for less than half a million dollars we will be able to create the next version of a truly high-fidelity haptic simulation where residents actually feel as if they are oper- ating. The cost for buying a single unit has not yet been determined, but it will definitely be affordable. As I mentioned, it is just a laptop and robotic arms and a small amount of software, so it will definitely be affordable at the university level.

Dr Paul Schipper (Portland, Ore). I think what you are doing is fantastic, and I applaud your efforts. Dr Grossi certainly knows more about computers than I ever will. When I look at these simulations though, they resemble the video games that I played in 1995 or earlier, early Castle Wolfenstein and DOOM, and when I see what my son is playing, Call of Duty Modern Warfare and Halo-3, it is like you are there in the video game. I am wondering what you think it will take for us to get to that level of realism in simulation, and I am also wondering if we get away from a PC platform and move to a Wii or an X-Box 360, would that be a different way to attack this?

Dr Solomon. Thank you for your point. Very good question and a question that comes up a lot. We need to compare this with what is reasonable, and to be honest, electronic arts developing games such as Call of Duty and Halo-3, the games I have played and enjoyed, are spending hundreds of millions of dollars and 20 years of research to do this. We will never get that kind of funding to spend hundreds of millions of dollars to do that. We try to make it as realistic looking as possible, but I do not foresee it ever reaching that point. We do hope to use their technology though and go as far as possible to make this realistic, but it will require a large sum of money to make these look realistic and a large amount of time and manpower. These companies tend to have thousands of people working on these video games at a time just for the graphic development.

Regarding your second question about integrating a personal video game system, it is definitely an option we have thought of. The PC-based system actually provides a little more manpower and workforce as far as the graphic simulation. It allows lowercost, higher-fidelity graphic simulation. It would be easier to have these at home, and currently we are looking at things like the built-in anatomy explorer that I mentioned to be visible on devices such as the iPad. This has all been developed in HTML5, which, without getting into too much detail, is visible on the $\mathrm{iPad}$, and students and residents and trainees would be able to download and explore the anatomy. Getting a haptic device to hook up to that would be expensive and probably insurmountably expensive for the individual trainees, but it is an idea that we have contemplated. 